\title{
Computational Analysis of Low-Density Hypersonic Flow over Backward-Facing Steps
}

\author{
Paulo H. M. Leite* and Wilson F. N. Santos ${ }^{\dagger}$ \\ National Institute for Space Research, Cachoeira Paulista, SP, 12630-000, BRAZIL
}

\begin{abstract}
This paper describes a numerical study on backward-facing steps situated in a rarefied hypersonic flow by employing the Direct Simulation Monte Carlo (DSMC) method. The work is motivated by the interest in investigating the step-height effect on the aerodynamic surface quantities. In this fashion, the primary aim of this paper is to examine the sensitivity of the heat transfer, pressure and skin friction coefficients with respect to stepheight variations. The analysis showed that the hypersonic flow past to a backward-facing step is characterized by a strong expansion around the step corner, which influences the aerodynamics surface properties downstream its back face. It was also found that changing the step height affected the aerodynamic surface quantities a distance of a few mean free paths upstream of it. Numerical results were compared with available experimental data of surface heat flux downstream of the step, showing a very reasonable agreement within experimental uncertainty.
\end{abstract}

\section{Nomenclature}

$a$

$A$

$C_{f}$

$C_{h}$

$C_{p}$

$c$

$d$

$D$

$F_{N}$

$h$

$H$

$K n$

l

L

$M$

$m$

$n$

$N$

$N_{f}$

$p$

$q$

$R e$

$R$

$T$

$u, v$

Speed of sound, m/s

Area, $\mathrm{m}^{2}$

Skin friction coefficient, Eq.( 5)

Heat transfer coefficient, Eq.( 1)

Pressure coefficient, Eq.( 3)

Molecular velocity, $\mathrm{m} / \mathrm{s}$

Molecular diameter, $\mathrm{m}$

Flat-plate total length, m

Ratio of real molecules to simulated molecules, dimensionless

Step height, $\mathrm{m}$

Dimensionless step height, $h / \lambda_{\infty}$

Knudsen number, $\lambda / l$

Characteristic length, m

Upper surface length, m

Mach number, $U / a$

Molecular mass, kg

Number density, $\mathrm{m}^{-3}$

Number of molecules

Dimensionless number flux, $N / n_{\infty} U_{\infty}$

Pressure, $\mathrm{N} / \mathrm{m}^{2}$

Heat flux, W/m²

Reynolds number, $U l / \mu$

Reentry capsule nose radius, $\mathrm{m}$

Temperature, $\mathrm{K}$

$U \quad$ Freestream velocity, $\mathrm{m} / \mathrm{s}$

* Graduate Student, Combustion and Propulsion Laboratory.

${ }^{\dagger}$ Researcher, Combustion and Propulsion Laboratory, and AIAA Member Grade. 


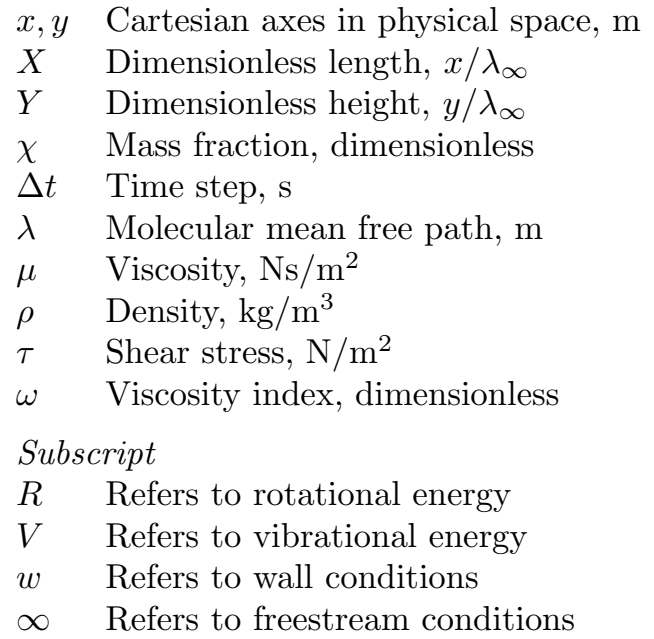

\section{Introduction}

$\mathrm{T}^{\mathrm{H}}$

HE development of aerospace technology has generated a strong demand on research associated with rarefied gas dynamics. Usually, the development of space vehicles in this area involves reentry type vehicles, exploratory interplanetary vehicles, etc. These space vehicles, which operate in the high atmosphere at hypersonic speeds, are generally designed with contour discontinuities, such as cavities, gaps or steps. The presence of these discontinuities in modern aerodynamics configurations occurs as a desired or undesired design feature, although a smooth aerodynamic shape of the surface is attempted.

The hypersonic flow over backward-facing steps involves flow separation and reattachment. The flow is characterized by a strong expansion wave around the step corner. In addition, it is known that the flow separation modifies the heat transfer at and beyond the separation region. Usually, the low velocity in the recirculation region is expected to cause relatively low heat-transfer rates. Nevertheless, at the reattachment zone, the heat-transfer rates may either increase gradually to their attached flow values or reach comparatively large values. As a result, the separating and reattaching flow phenomena may affect locally thermal and aerodynamic loads, which may exceed the ones of a smooth surface. Therefore, in order to operate safely, detailed knowledge of these loads are necessary.

Many experimental and theoretical studies ${ }^{1,2,3,4,5,6,7,8,9,10,11,12,13,14,15}$ have been conducted in order to understand the physical aspects of a hypersonic flow past to this type of discontinuities, characterized by a sudden change on the surface slope. For the purpose of this introduction, it will be sufficient to describe only a few of these studies.

Data presented by Charwat et al. ${ }^{1}$ indicated that flow which separates from an isolated backward-facing step impinges on the wall approximately a distance of seven times the step height downstream of the step if the boundary layer is laminar, and approximately five times the step height downstream for a turbulent boundary layer.

Rom and Seginer ${ }^{2}$ investigated experimentally the heat-transfer rate on a 2-D backward-facing step in a laminar supersonic flow, corresponding to Mach number in the range of 1.5 to 2.5, and Reynolds number in the range of $10^{3}$ to $10^{5}$. Results indicated that heat-transfer rates changed with the distance behind the step. In addition, it was found that the heat transfer rates depended on the ratio of the boundary-layer thickness at the separation to the step height.

Gai and Milphorpe ${ }^{9}$ presented experimental and computational results of a high enthalpy flow over a blunted-stepped cone. Basically, an axisymmetric backward-facing step of height of $3 \mathrm{~mm}$ and $6 \mathrm{~mm}$ located at a distance of $101 \mathrm{~mm}$ from the nose. The analysis showed that the heat transfer rate was typical of that in a separated flow, i.e., a sudden fall in heat transfer very near the step and then a gradual increase beyond it. The experimental data showed a decrease in heat transfer rate after reattachment, whereas the numerical prediction exhibited a plateau for a considerable distance.

Grotowsky and Ballmann ${ }^{11}$ investigated laminar hypersonic flow over forward- and backward-facing steps by employing Navier-Stokes equations. The hypersonic flow over the steps were simulated by considering freestream Mach number of 8, Reynolds number of the order of $10^{8}$ and an altitude of $30 \mathrm{~km}$. According to them, the computational results presented a good agreement with experimental data available in the 
literature. They also pointed out that the quantitative comparison exhibited major differences for the wall heat flux, probably due to the difficult in how to measure accurately.

A numerical study on backward-facing steps, situated in a rarefied hypersonic flow, has been examined by Leite and Santos ${ }^{13}$ by employing the DSMC method. The work was motivated by the interest in investigating the step height effect on the flowfield structure. The primary emphasis was to examine the sensitivity of velocity, density, pressure and temperature fields with respect to step-height variations of such backwardfacing steps. The analysis showed that the hypersonic flow past a backward-facing step was characterized by a strong expansion wave around de corner of the step, which influenced the downstream separation region. It was found that the recirculation region relies on the back-face height. The analysis also showed that disturbances downstream the step depended on changes in the back-face height of the steps.

In continuation of the backward-facing step study, the purpose of the present account is to extend further the previous analysis ${ }^{13}$ by investigating the impact of the back-face height on the aerodynamic surface quantities. In this scenario, the primary goal of this paper is to assess the sensitivity of the heat transfer, pressure, and skin friction coefficients due to variations on the back-face height of the step. The focus of the present study is the low-density region in the upper atmosphere. At high altitude, and therefore, low density environment, the molecular collision rate is low and the energy exchange occurs under nonequilibrium conditions. As a result, the degree of molecular non-equilibrium is such that the Navier-Stokes equations are inappropriate. In such a circumstance, the Direct Simulation Monte Carlo (DSMC) method will be employed to calculate the hypersonic two-dimensional flow over a backward-facing step.

\section{Computational Method and Procedure}

In order to study rarefied flow with a significant degree of non-equilibrium, the Direct Simulation Monte Carlo (DSMC) method ${ }^{16}$ is usually employed. The DSMC method has become the most common computational technique for modeling complex flows of engineering interest in the transitional flow regime. The DSMC method models a gas flow by using a computer to track the trajectory of simulated particles, where each simulated particle represents a fixed number of real gas particles. The simulated particles are allowed to move and collide, while the computer stores their positions, velocities and other physical properties such as internal energy.

In the present account, molecular collisions are modeled by using the variable hard sphere (VHS) molecular model ${ }^{17}$ and the no time counter (NTC) collision sampling technique. ${ }^{18}$ Energy exchange between kinetic and internal modes is controlled by the Borgnakke-Larsen statistical model. ${ }^{19}$ Simulations are performed using a non-reacting gas model consisting of $76.3 \%$ of $\mathrm{N}_{2}$ and $23.7 \%$ of $\mathrm{O}_{2}$. Energy exchanges between the translational and internal modes, rotational and vibrational, are considered. The probability of an inelastic collision determines the rate at which energy is transferred between the translational and internal modes after an inelastic collision. For a given collision, the probability is defined by the inverse of the number of relaxation, which corresponds to the number of collisions needed, on average, for a molecule to undergo relaxation. The rates of rotational and vibrational relaxation are dictated by collision numbers $Z_{R}$ and $Z_{V}$, respectively. In the present account, rotational $Z_{R}$ and vibrational $Z_{V}$ collision numbers were obtained in a collision energy-based procedure, as suggested by Boyd ${ }^{20}$ for rotation and by Bird ${ }^{21}$ for vibration.

\section{Geometry Definition}

In the study being reported herein, discontinuities present on the surface of a reentry capsule are modeled by a backward-facing step. By assuming that the back face $h$ is much smaller than the nose radius $R$ of a reentry capsule, i.e., $h / R \ll 1$, then the hypersonic flow over the step may be considered as a hypersonic flow over a flat plate with a backward-facing step. Figure 1 illustrates a schematic view of the model employed and presents the important parameters.

According to Fig. $1, M_{\infty}$ represents the freestream Mach number, $h$ the back-face height, $L$ the length of the step upstream surface, and $D$ the total length of the flat plate. It was considered that the flat plate is infinitely long but only the total length $D$ is examined. It was assumed a back-face height $h$ of 3,6 , and 9 $\mathrm{mm}$, which correspond to $H\left(=h / \lambda_{\infty}\right)$ of $3.23,6.46$, and 9.69 , respectively, where $\lambda_{\infty}$ is the freestream mean free path. In addition, it was assumed $L / \lambda_{\infty}$ of 50 and $D / \lambda_{\infty}$ of 150,170 and 190 for $H$ of 3.23, 6.46, and 9.69 , respectively.

In order to bring out the essential features of the back-face height effects on the aerodynamic surface 


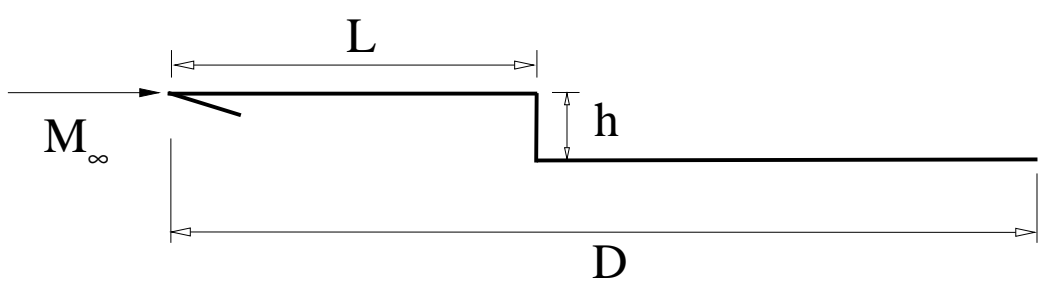

Figure 1. Drawing illustrating the backward-facing step.

properties, it becomes imperative to compare the flowfield behavior of a flat plate with a back-forward step with that without a step. In this manner, a flat plate without a step works as a benchmark for the cases with a step, and will be referred herein as the flat-plate case.

\section{Freestream and Flow Conditions}

Freestream conditions and gas properties employed in the present calculations are those given by Leite and Santos ${ }^{13}$ and tabulated in Tabs. 1 and 2, respectively. These flow conditions represent those experienced by a Brazilian capsule, named SARA (acronyms for SAtélite de Reentrada Atmosférica) at an altitude of 70 $\mathrm{km}$. Referring to Tables 1 and $2, T_{\infty}, p_{\infty}, \rho_{\infty}, \mu_{\infty}, n_{\infty}, \lambda_{\infty}$, and $U_{\infty}$ stand, respectively, for temperature, pressure, density, viscosity, number density, molecular mean free path, and velocity, and $\chi, m, d$ and $\omega$ account, respectively, for mass fraction, molecular mass, molecular diameter and viscosity index.

The freestream velocity $U_{\infty}$, assumed to be constant at $7456 \mathrm{~m} / \mathrm{s}$, corresponds to a freestream Mach number $M_{\infty}$ of 25 . The wall temperature $T_{w}$ is assumed constant at $880 \mathrm{~K}$. This temperature is chosen to be representative of the surface temperature near the stagnation point of a reentry capsule and is assumed to be uniform over the backward-facing step. It is important to mention that the surface temperature is low compared to the stagnation temperature of the air. This assumption seems reasonable since practical surface materials will probably be destroyed if surface temperature is allowed to approach the stagnation temperature.

By assuming the back-face height $h$ as the characteristic length, the Knudsen number $K n_{h}$ corresponds to $0.3095,0.1548$ and 0.1032 for height $h$ of 3,6 and $9 \mathrm{~mm}$, respectively. Finally, the Reynolds number $R_{h}$, also based on the back-face height $h$ and on conditions in the undisturbed stream, is around 136, 272, and 409 for height $h$ of 3,6 and $9 \mathrm{~mm}$, respectively.

Table 1. Freestream flow conditions

\begin{tabular}{ccccccc}
\hline \hline Altitude $(\mathrm{km})$ & $T_{\infty}(\mathrm{K})$ & $p_{\infty}\left(\mathrm{N} / \mathrm{m}^{2}\right)$ & $\rho_{\infty}\left(\mathrm{kg} / \mathrm{m}^{3}\right)$ & $\mu_{\infty}\left(\mathrm{Ns} / \mathrm{m}^{2}\right)$ & $n_{\infty}\left(\mathrm{m}^{-3}\right)$ & $\lambda_{\infty}(\mathrm{m})$ \\
\hline 70 & 220.0 & 5.582 & $8.753 \times 10^{-5}$ & $1.455 \times 10^{-5}$ & $1.8209 \times 10^{21}$ & $9.03 \times 10^{-4}$ \\
\hline \hline
\end{tabular}

Table 2. Gas properties

\begin{tabular}{ccccc}
\hline \hline & $\chi$ & $m(\mathrm{~kg})$ & $d(\mathrm{~m})$ & $\omega$ \\
\hline $\mathrm{O}_{2}$ & 0.237 & $5.312 \times 10^{-26}$ & $4.01 \times 10^{-10}$ & 0.77 \\
$\mathrm{~N}_{2}$ & 0.763 & $4.650 \times 10^{-26}$ & $4.11 \times 10^{-10}$ & 0.74 \\
\hline \hline
\end{tabular}




\section{Computational Flow Domain and Grid}

A schematic view of the computational domain is depicted in Fig. 2. The computational domain is divided into twelve regions (R1 to R12), which are subdivided into computational cells. The cells are further subdivided into subcells, two subcells/cell in each coordinate direction. The cell provides a convenient reference for the sampling of the macroscopic gas properties, while the collision partners are selected from the same subcell for the establishment of the collision rate.

According to Fig. 2, side I-A is defined by the backward-facing step surface. Diffuse reflection with complete thermal accommodation is the condition applied to this side. Side I-B is a plane of symmetry, where all flow gradients normal to the plane are zero. At the molecular level, this plane is equivalent to a specular reflecting boundary. Sides II and III are the freestream side through which simulated molecules enter and exit. Side II is positioned at $5 \lambda_{\infty}$ upstream of the upper-surface leading edge, and side III defined at $30 \lambda_{\infty}$ above the upper surface. Finally, the flow at the downstream outflow boundary, side IV, is predominantly supersonic and vacuum condition is specified. ${ }^{22}$ At this boundary, simulated molecules can only exit. Nevertheless, it should be remarked that, close to the wall, molecules may not be moving at supersonic speed. As a result, in this subsonic region close to the wall, there is an interaction between the flow and the downstream boundary. However, the extent of the upstream effect of this boundary condition can be determined by changing the length of the lower surface. For the conditions investigated in the present account, the upstream disturbance is approximately of $10 \lambda_{\infty}$, as can be observed in the results presented in the subsequent sections.

The numerical accuracy in DSMC method depends on the cell size chosen, on the time step as well as on the number of particles per computational cell. In the DSMC code, the linear dimensions of the cells should be small in comparison with the scale length of the macroscopic flow gradients normal to the streamwise directions, which means that the cell dimensions should be the order of or smaller than the local mean free path. ${ }^{23,24}$ The time step should be chosen to be sufficiently small in comparison with the local mean collision time..$^{25,26}$ In general, the total simulation time, discretized into time steps, is based on the physical time of the real flow. Finally, the number of simulated particles has to be large enough to make statistical correlations between particles significant. These effects were investigated in order to determine the number of cells and the number of particles required to achieve grid independent solutions.

As part of the verification process, a grid independence study was made with three different structured meshes - coarse, standard and fine - in each coordinate direction. The effect of altering the cell size in the $x$ - and $y$-directions was investigated for a coarse and fine grids with, respectively, $50 \%$ less and 100\% more cells with respect to the standard grid. Solutions (not shown) were nearly identical for all grids used and were considered fully grid independent. Table 3 summarizes the main characteristics for the standard grid related to twelve regions (R1 to R12 in Fig. 2) for the back-face height $H$ of 3.23, 6.46, and 9.69. In this context, for $H$ of $3.23,6.46$, and 9.69, the total number of cells correspond, respectively, to $26,800,33,400$, and 39,460 cells.

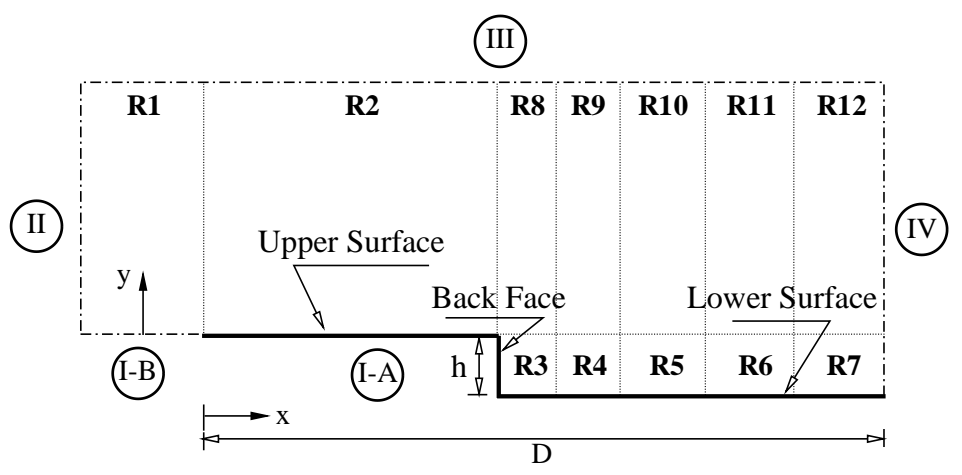

Figure 2. Drawing illustrating the computational domain. 
Table 3. Region Dimensions $(x \times y)$ and number of cells $[x \times y]$ for cases $H$ of 3.23, 6.46, and 9.69.

\begin{tabular}{lccc}
\hline \hline & $\mathbf{H}=\mathbf{3 . 2 3}$ & $\mathbf{H}=\mathbf{6 . 4 6}$ & $\mathbf{H}=\mathbf{9 . 6 9}$ \\
\hline R1 & $\left(5 \lambda_{\infty} \times 30 \lambda_{\infty}\right)[10 \times 50]$ & $\left(5 \lambda_{\infty} \times 30 \lambda_{\infty}\right)[10 \times 50]$ & $\left(5 \lambda_{\infty} \times 30 \lambda_{\infty}\right)[10 \times 50]$ \\
R2 & $\left(50 \lambda_{\infty} \times 30 \lambda_{\infty}\right)[120 \times 60]$ & $\left(50 \lambda_{\infty} \times 30 \lambda_{\infty}\right)[120 \times 60]$ & $\left(50 \lambda_{\infty} \times 30 \lambda_{\infty}\right][120 \times 60]$ \\
R3 & $\left(10 \lambda_{\infty} \times 3.23 \lambda_{\infty}\right)[30 \times 30]$ & $\left(12 \lambda_{\infty} \times 6.46 \lambda_{\infty}\right)[30 \times 50]$ & $\left(14 \lambda_{\infty} \times 9.69 \lambda_{\infty}\right)[36 \times 70]$ \\
R4 & $\left(15 \lambda_{\infty} \times 3.23 \lambda_{\infty}\right)[30 \times 20]$ & $\left(18 \lambda_{\infty} \times 6.46 \lambda_{\infty}\right)[30 \times 30]$ & $\left(21 \lambda_{\infty} \times 9.69 \lambda_{\infty}\right)[36 \times 40]$ \\
R5 & $\left(25 \lambda_{\infty} \times 3.23 \lambda_{\infty}\right)[30 \times 20]$ & $\left(30 \lambda_{\infty} \times 6.46 \lambda_{\infty}\right)[30 \times 30]$ & $\left(35 \lambda_{\infty} \times 9.69 \lambda_{\infty}\right)[36 \times 40]$ \\
R6 & $\left(25 \lambda_{\infty} \times 3.23 \lambda_{\infty}\right)[30 \times 20]$ & $\left(30 \lambda_{\infty} \times 6.46 \lambda_{\infty}\right)[30 \times 30]$ & $\left(35 \lambda_{\infty} \times 9.69 \lambda_{\infty}\right)[36 \times 40]$ \\
R7 & $\left(25 \lambda_{\infty} \times 3.23 \lambda_{\infty}\right)[30 \times 20]$ & $\left(30 \lambda_{\infty} \times 6.46 \lambda_{\infty}\right)[30 \times 30]$ & $\left(35 \lambda_{\infty} \times 9.69 \lambda_{\infty}\right)[36 \times 40]$ \\
R8 & $\left(10 \lambda_{\infty} \times 30 \lambda_{\infty}\right)[30 \times 60]$ & $\left(12 \lambda_{\infty} \times 30 \lambda_{\infty}\right)[40 \times 60]$ & $\left(14 \lambda_{\infty} \times 30 \lambda_{\infty}\right)[46 \times 60]$ \\
R9 & $\left(15 \lambda_{\infty} \times 30 \lambda_{\infty}\right)[30 \times 70]$ & $\left(18 \lambda_{\infty} \times 30 \lambda_{\infty}\right)[40 \times 70]$ & $\left(21 \lambda_{\infty} \times 30 \lambda_{\infty}\right)[46 \times 70]$ \\
R10 & $\left(25 \lambda_{\infty} \times 30 \lambda_{\infty}\right)[50 \times 70]$ & $\left(30 \lambda_{\infty} \times 30 \lambda_{\infty}\right)[70 \times 70]$ & $\left(35 \lambda_{\infty} \times 30 \lambda_{\infty}\right)[80 \times 70]$ \\
R11 & $\left(25 \lambda_{\infty} \times 30 \lambda_{\infty}\right)[60 \times 70]$ & $\left(30 \lambda_{\infty} \times 30 \lambda_{\infty}\right)[70 \times 70]$ & $\left(35 \lambda_{\infty} \times 30 \lambda_{\infty}\right)[80 \times 70]$ \\
R12 & $\left(25 \lambda_{\infty} \times 30 \lambda_{\infty}\right)[60 \times 70]$ & $\left(30 \lambda_{\infty} \times 30 \lambda_{\infty}\right)[80 \times 70]$ & $\left(35 \lambda_{\infty} \times 30 \lambda_{\infty}\right)[90 \times 70]$ \\
\hline \hline
\end{tabular}

A discussion of the verification process, cell size, time step, and number of molecules effects on the aerodynamic surface quantities for the backward-facing steps presented herein is described in detail in Leite. ${ }^{27}$ Furthermore, as part of the validation process, results for density, velocity and translational temperature were compared with those obtained from other established DSMC code and experimental data in order to ascertain how well the DSMC code employed in this study is able to predict hypersonic flow in a flat plate. Details of this comparison is also presented in Leite. ${ }^{27}$

\section{Computational Results and Discussion}

This section focuses on the effects that take place in the aerodynamic surface quantities due to variations on the back-face height of a backward-facing step. Aerodynamic surface quantities of particular interest in the transition flow regime are number flux, heat transfer, pressure, and skin friction. In this scenario, this section discusses and compares differences in these quantities expressed in a dimensionless coefficient form.

\section{A. Number Flux}

The number flux $N$ is calculated by sampling the molecules impinging on the surface by unit time and unit area. The distribution of the number flux along the step surface - upper, face and lower - is illustrated in Fig. 3 as a function of the dimensionless step height $H$. In this group of plots, $N_{f}$ represents the number flux $N$ normalized by $n_{\infty} U_{\infty}$, where $n_{\infty}$ is the freestream number density and $U_{\infty}$ is the freestream velocity. In addition, $X$ and $Y$ are the lengths $x$ and $y$ normalized by the freestream mean free path $\lambda_{\infty}$. As a basis of comparison, the dimensionless number flux for the flat-plate case, i.e., a flat plate without a step, is also illustrated in the plots.

According to Fig. 3(a), it is observed that, from the leading edge up to the vicinity of the step corner, the number flux behavior for the flat plate with a backward-facing step is similar to that one without a step. This is an expected behavior, since the flowfield in this region has no idea about the presence of the step. Nevertheless, very close to the step corner, the number flux drops off due to the flow expansion around the step corner. After that, at the vicinity of the back face on the lower surface, the number flux suddenly goes to zero and afterwards increases significantly as the flow develops along the lower surface. In addition, a dramatically reduction in the number flux is observed at the end of the upper surface. The reason for that is associated with the vacuum condition assumed for the downstream outflow boundary, side IV, as explained earlier.

Referring to Fig. 3(b), it is seen that the maximum number flux to the back face of the step is an order of magnitude smaller than those values observed for the upper and lower surfaces. This behavior is directly related to the recirculation region downstream of the back face. It should be mentioned in this context that, the recirculation region at the vicinity of the back face is a region of low density. ${ }^{13}$ As a result, the number flux to the face is very low. Similar to that one showed to the lower surface, the number flux to the face is 


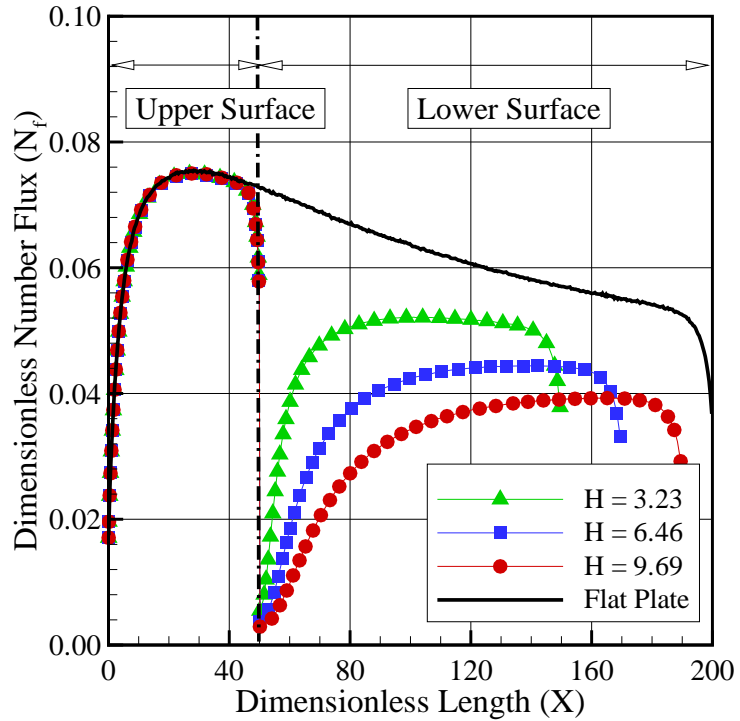

(a) Upper and lower surfaces

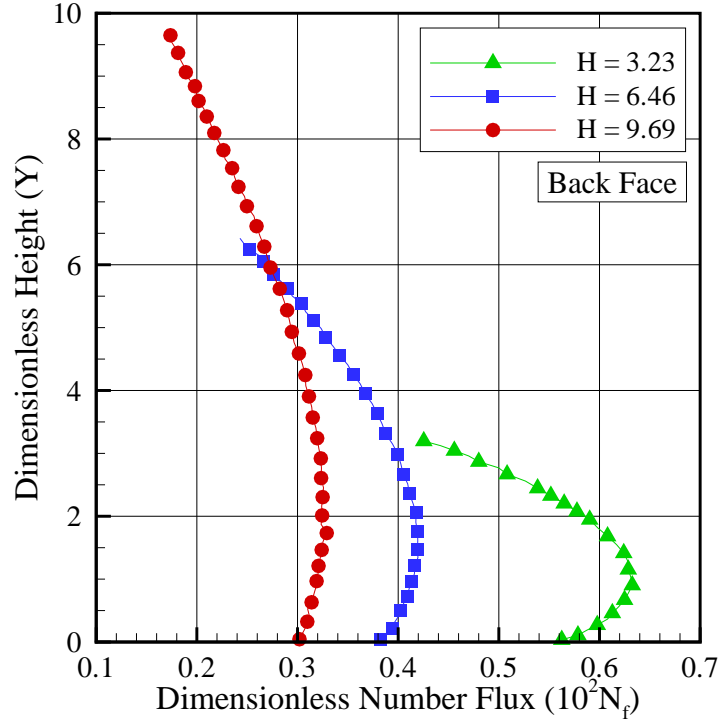

(b) Back face

Figure 3. Distribution of the number flux $N_{f}$ along (a) the upper and lower surfaces and along the (b) back face as function of the step height $h$.

a function of step height $h$, i.e, it decreases with the step-height rise.

\section{B. Heat Transfer Coefficient}

The heat transfer coefficient $C_{h}$ is defined as follows,

$$
C_{h}=\frac{q_{w}}{\frac{1}{2} \rho_{\infty} U_{\infty}^{3}}
$$

where the heat flux $q_{w}$ to the body surface is calculated by the net energy flux of the molecules impinging on the surface. A flux is regarded as positive if it is directed toward the body surface. The net heat flux $q_{w}$ is related to the sum of the translational, rotational and vibrational energies of both incident and reflected molecules, and defined by,

$$
q_{w}=q_{i}-q_{r}=\frac{F_{N}}{A \Delta t}\left\{\sum_{j=1}^{N}\left[\frac{1}{2} m_{j} c_{j}^{2}+e_{R j}+e_{V j}\right]_{i}-\sum_{j=1}^{N}\left[\frac{1}{2} m_{j} c_{j}^{2}+e_{R j}+e_{V j}\right]_{r}\right\}
$$

where $F_{N}$ is the number of real molecules represented by a single simulated molecule, $\Delta t$ is the time step, $A$ the area, $N$ is the number of molecules colliding with the surface by unit time and unit area, $m$ is the mass of the molecules, $c$ is the velocity of the molecules, $e_{R}$ and $e_{V}$ stand for rotational and vibrational energies, respectively. Subscripts $i$ and $r$ refer to incident and reflect molecules.

The dependence of the heat transfer coefficient $C_{h}$ on the back-face height $h$ is displayed in Figs. 4(a,b) for the upper, face, and lower surfaces. Again, for comparative purposes, the heat transfer coefficient for the flat plate without a step is also illustrated in the plots. According to Fig. 4(a), it is clearly noticed that, except very close to the step corner, the heat transfer coefficient to the upper surface follows the same tendency of that presented by the number flux in the sense that it is not affected by the presence of the step. Along the lower surface, the minimum heat transfer coefficient occurs at the vicinity of the back face in the recirculation region. After that, the heat transfer coefficient increases to a maximum value after the 


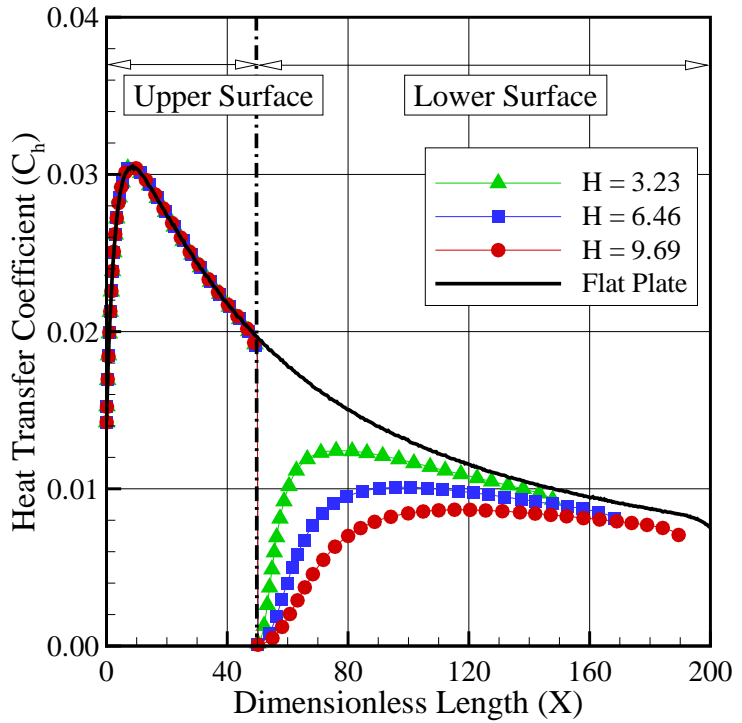

(a) Upper and lower surfaces

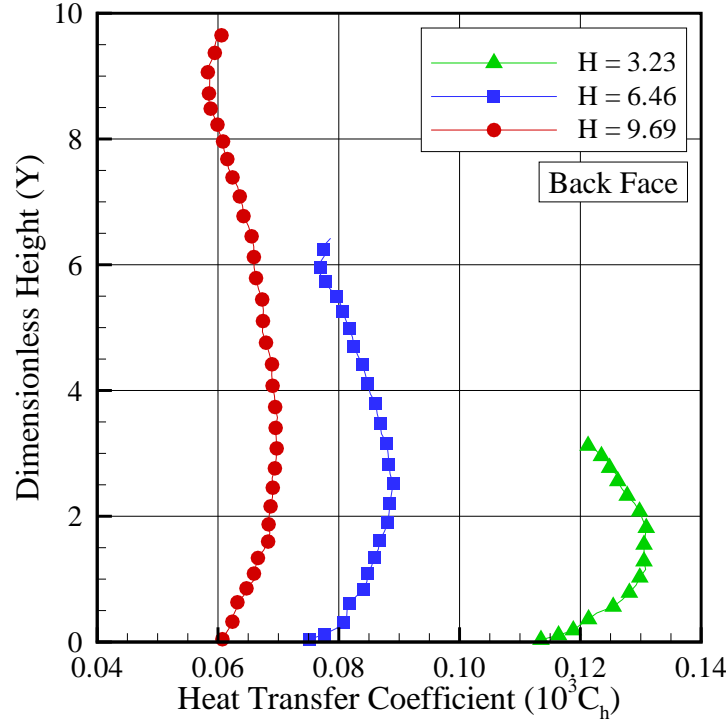

(b) Back face

Figure 4. Distribution of heat transfer coefficient $C_{h}$ along (a) the upper and lower surfaces and along the (b) back face as function of the step height $h$.

flow reattachment. In the following, $C_{h}$ decays and approaches the level observed for the flat plate without a step. It is important to mention that, after the flow reattachment, the flowfield behavior is similar to that along a flat plate. In addition, no shock-wave formation was observed on the lower surface, for the conditions investigated, as shown by Leite and Santos. ${ }^{13}$

Based on Fig. 4(b), along the back face, the heat transfer coefficient values are two order of magnitude lower than those observed on the upper or lower surfaces. As mentioned earlier, this is due to the recirculation region formed at the vicinity of the back face. In this region, density $\rho$ is lower that that of the freestream density $\rho_{\infty}$, the velocity of the molecules is very low as compared to the freestream velocity $U_{\infty}$, and the temperature is approximately the same as the wall temperature $T_{w} \cdot{ }^{13}$ As a result, there is a balance between the incident heat flux $q_{i}$ and the reflected heat flux $q_{r}$ contributions, as defined by Eq. 2, since molecules in this region have low energy.

\section{Pressure Coefficient}

The pressure coefficient $C_{p}$ is defined as follows,

$$
C_{p}=\frac{p_{w}-p_{\infty}}{\frac{1}{2} \rho_{\infty} U_{\infty}^{2}}
$$

where the pressure $p_{w}$ on the body surface is calculated by the sum of the normal momentum fluxes of both incident and reflected molecules at each time step as follows,

$$
p_{w}=p_{i}-p_{r}=\frac{F_{N}}{A \Delta t} \sum_{j=1}^{N}\left\{\left[(m v)_{j}\right]_{i}-\left[(m v)_{j}\right]_{r}\right\}
$$

where $v$ is the velocity component of the molecule $j$ in the surface normal direction.

The variation of the pressure coefficient $C_{p}$ caused by changes in the back-face height $h$ is demonstrated in Figs. 5(a,b) for upper, face, and lower surfaces. According to these figures, it is clearly noted that pressure 


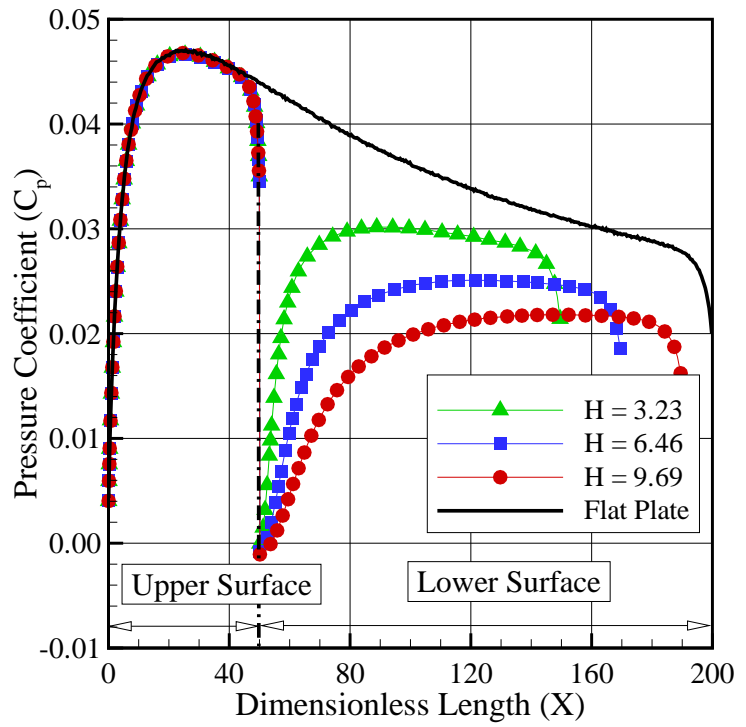

(a) Upper and lower surfaces

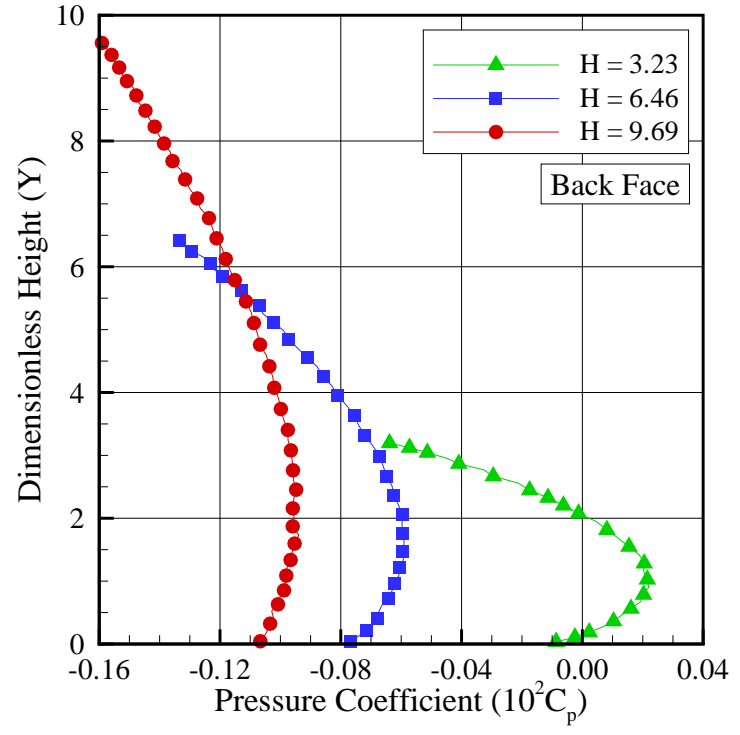

(b) Back face

Figure 5. Distribution of pressure coefficient $C_{p}$ along (a) the upper and lower surfaces and along the (b) back face as function of the step height $h$.

coefficient behavior follows the same trend shown by the number flux in the sense that: (1) from the leading edge up to the the vicinity of the step corner, the pressure coefficient behavior for the flat plate with a backward-facing step is similar to that one without a step, (2) the presence of the backward-facing step affects the pressure coefficient in a small region very close to the step corner, (3) in this small region, the pressure coefficient presents a sudden decrease as compared to the pressure coefficient for the flat plate without a step, (4) the smallest values for the pressure coefficient takes place along the back face of the step, (5) in this region, the wall pressure $p_{w}$ is lower than the freestream pressure $p_{\infty}$. Similar to the number flux and to the heat transfer coefficient, the smallest values on the pressure coefficient are directly related to the recirculation region, as pointed out earlier.

\section{Skin Friction Coefficient}

The skin friction coefficient $C_{f}$ is defined as follows,

$$
C_{f}=\frac{\tau_{w}}{\frac{1}{2} \rho_{\infty} U_{\infty}^{2}}
$$

where the shear stress $\tau_{w}$ on the body surface is calculated by the sum of the tangential momentum fluxes of both incident and reflected molecules impinging on the surface at each time step by the following expression,

$$
\tau_{w}=\tau_{i}-\tau_{r}=\frac{F_{N}}{A \Delta t} \sum_{j=1}^{N}\left\{\left[(m u)_{j}\right]_{i}-\left[(m u)_{j}\right]_{r}\right\}
$$

where $u$ is the velocity component of the molecule $j$ in the surface tangential direction.

It should be mentioned that, for the gas-surface interaction model adopted herein, diffusion reflection, reflected molecules have a tangential moment equal to zero, since the molecules essentially lose, on average, their tangential velocity components. In a diffuse reflection, the molecules are reflected equally in all directions, and the final velocity of the molecules is randomly assigned according to a half-range Maxwellian 


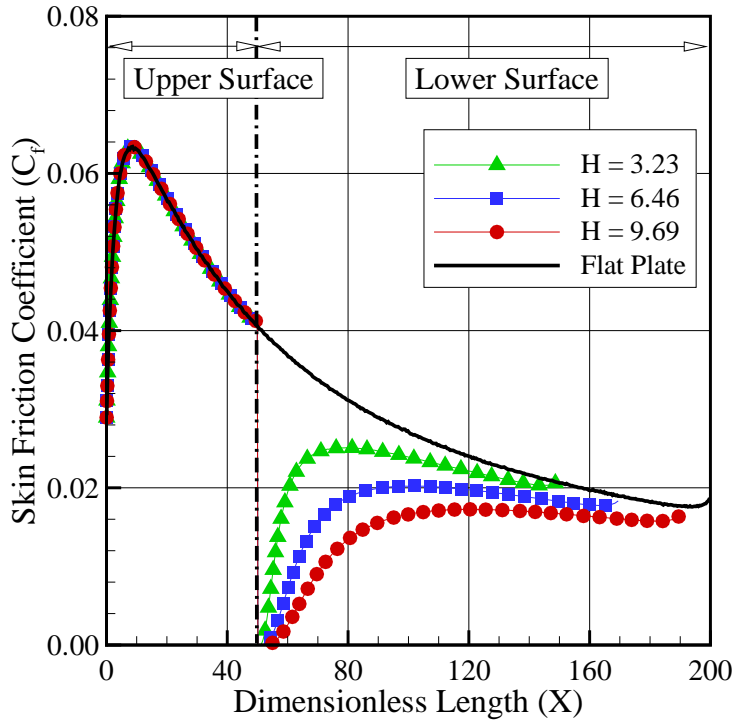

(a) Upper and lower surfaces

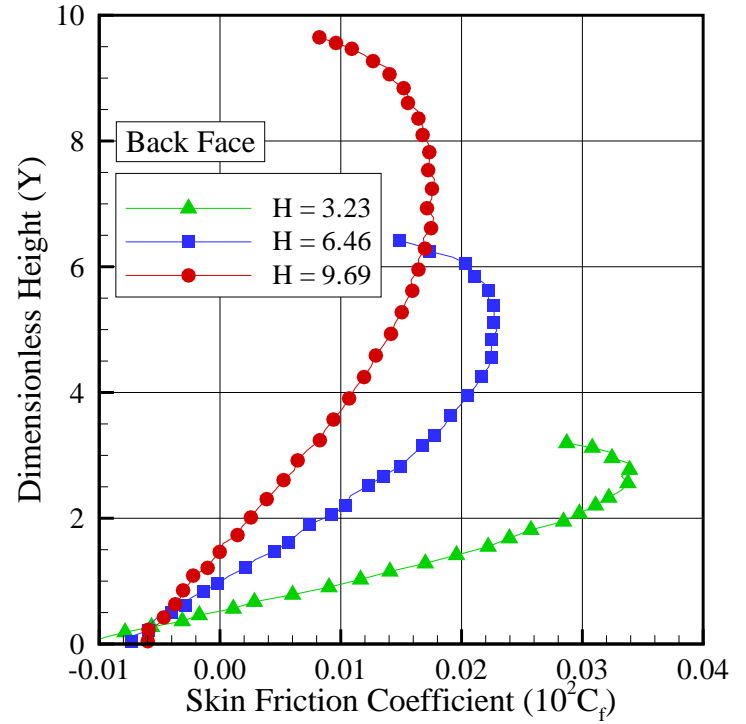

(b) Back face

Figure 6. Distribution of skin friction coefficient $C_{f}$ along (a) the upper and lower surfaces and along the (b) back face as function of the step height $h$.

distribution determined by the wall temperature. In this fashion, the contribution of the reflected tangential momentum flux $\tau_{r}$ that appears in Eq. 6 is equal to zero. Nevertheless, for incomplete surface accommodation, the reflected tangential momentum flux $\tau_{r}$ contributes to the skin friction coefficient.

The influence of the back-face height $h$ in the skin friction coefficient $C_{f}$ is depicted in Figs. 6(a,b) for upper, face, and lower surfaces. Looking first to Fig. 6(a), it is observed that, in general, the skin friction coefficient behavior is similar to those for the other surface quantities. Along the lower surface, the skin friction coefficient is negative near to the face of the step and becomes positive at the reattachment point. After that, the skin friction coefficient increases and reaches the values observed for the flat plate without steps. The condition $C_{f}=0$ or $\tau_{w}=0$, usually used to define the reattachment point, ${ }^{10}$ takes place at $X$ $=50.51,51.25$ e 52.40 for dimensionless back-face height $H$ of $3.23,6.46$, and 9.69 , respectively. Turning next to Fig. 6(b), it is seen that the skin friction coefficient $C_{f}$ along the step face is at least two orders of magnitude smaller than that observed along the upper surface.

\section{E. Comparison with Experimental Data}

In this section, the behavior of the heat flux to the backward-facing step surface is compared to experimental data from experiments conducted by Wada and Inoue, ${ }^{6}$ Gai et al., ${ }^{7}$ and Hayne et al. ${ }^{12}$ These experiments are related to hypersonic laminar flow over backward-facing steps. In these experiments, the flow enthalpy ranged from $1.5 \mathrm{MJ} / \mathrm{kg}$ to $26 \mathrm{MJ} / \mathrm{kg}$, covering from undissociated to moderately dissociated freestream. In addition, Wada and Inoue ${ }^{6}$ conducted their experiments in a hypersonic gun tunnel in a free stream at Mach number around 10 generated by a conical nozzle. Gai et al. ${ }^{7}$ considered a two-dimensional backward-facing step in a hypersonic high enthalpy flow. Their tests were conducted in a free-piston-driven shock tunnel T3 with air as test gas and Helium as driver gas, with Mach number from 7.7 to 10. Finally, Hayne et al. ${ }^{12}$ conducted their experiments in a X2 expansion tube and in a T4 shock tunnel, by using air as a working fluid with Mach number from 6.3 to 7 . Table 4, adapted from Gai and Hayne, ${ }^{14}$ summarizes the important conditions related to these experiments. In this table, $h_{o}$ is the stagnation enthalpy, $R e$ refers to Reynolds number, $\gamma$ stands for the ratio of the specific heats, $\bar{V}_{\infty}$ represents the viscous interaction parameter, and, finally, $\Omega$ the Damkohler number.

The distribution of the heat flux to the backward-facing step surface is demonstrated in Fig. 7 in terms 
Table 4. Flow conditions (adapted from Gai and Hayne ${ }^{14}$ )

\begin{tabular}{lcccccc}
\hline \hline Reference & $h_{o}(\mathrm{MJ} / \mathrm{kg})$ & $R e / m$ & $\gamma$ & $M_{\infty}$ & $\bar{V}_{\infty}$ & $\Omega$ \\
\hline Wada and Inoue $^{6}$ & 1.5 & $36-50 \times 10^{-5}$ & 1.4 & 10.4 & 0.016 & 0.0 \\
Gai et al. & 2.6 & $46.6 \times 10^{-5}$ & 1.4 & 10.1 & 0.019 & 0.0083 \\
Hayne et al. $^{12}(\mathrm{a})$ & 26.0 & $11.2 \times 10^{-5}$ & 1.35 & 7.0 & 0.029 & 0.133 \\
Hayne et al. $^{12}(\mathrm{~b})$ & 5.4 & $16.9 \times 10^{-5}$ & 1.38 & 6.2 & 0.027 & 0.0569 \\
Hayne et al. $^{12}(\mathrm{c})$ & 5.6 & $58.5 \times 10^{-5}$ & 1.37 & 6.1 & 0.015 & 0.0596 \\
\hline \hline
\end{tabular}

of the Stanton number, $S t$. In this plots, data are expressed in terms of Stanton number ratio, $S t / S t_{f p}$, where $S t_{f p}$ is the Stanton number for the flat-plate case at the same location. In addition, $X_{h}^{\prime}$ is the distance downstream of the step, $x-L$, normalized by the step height $h$. On examining Fig. 7, it is seen that Stanton number ratio $S t / S t_{f p}$ for DSMC simulations present a very reasonable agreement with those obtained from experiments. It is important to recall that experimental data shown in Fig. 7 were base on different tests with widely differing freestream conditions, as tabulated in Tab. 4.

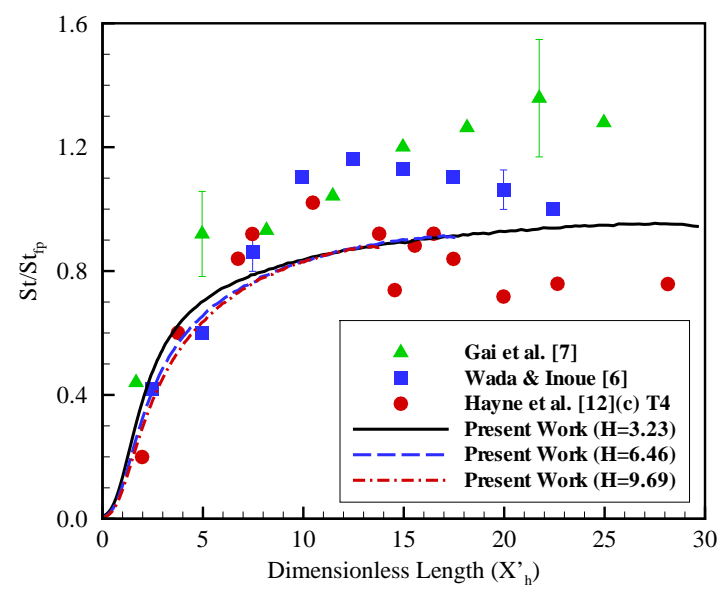

(a)

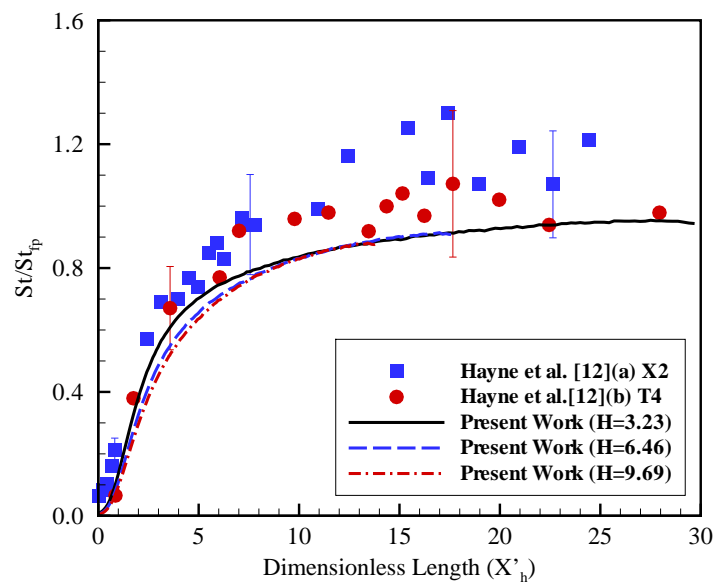

(b)

Figure 7. Distribution of Stanton number ratio $\left(S t / S t_{f p}\right)$ downstream of a backward-facing step.

\section{Concluding Remarks}

Computations of a rarefied hypersonic flow on backward-facing steps have been performed by using the Direct Simulation Monte Carlo (DSMC) method. The calculations provided information concerning the behavior of the aerodynamic surface quantities on backward-facing steps. Effects of the back-face height on the number flux, heat transfer, pressure and skin friction coefficients were investigated for a representative range of parameters. The back-face height ranged from 3 to $9 \mathrm{~mm}$, which corresponded Knudsen numbers in the transition flow regime.

The analysis showed that the aerodynamic surface properties were affected a distance of a few mean free paths upstream of the step location. It was found that the heat flux to the surface as well as the wall pressure increased along the lower surface downstream the back face. Nevertheless, the heat flux rise and wall pressure rise were below those values for a flat plate without a step. Comparison with experimental 
data showed that numerical results presented a very reasonable agreement within experimental uncertainty.

\section{Acknowledgments}

The authors would like to acknowledge the financial support for this research, provided by CNPq (Conselho Nacional de Desenvovimento Cientifico e Tecnologico) under Grant No. 473267/2008-0, and the financial support in order to attend this conference, provided by Capes (Coordenação de Aperfeiçoamento de Pessoal de Nível Superior) under Grant No. 2848/13-9.

\section{References}

${ }^{1}$ Charwat, A. F., Dewey, C. F., Roos, J. N. and Hitz, J. A., "An Investigation of Separated Flows. 1: The Pressure Field", Journal of aerospace Sciences, Vol. 28, No. 6, 1961, pp. 457-470.

${ }^{2}$ Rom, J., and Seginer, A., "Laminar Heat Transfer to a two-Dimensional Backward Facing Step from the High-Enthalpy Supersonic Flow in the Shock Tube", AIAA Journal, Vol. 2, No. 2, 1964, pp. 251-255.

${ }^{3}$ Scherberg, M. G., and Smith, H. E., "An Experimental Study of Supersonic Flow over a Rearward Facing Step", $A I A A$ Journal, Vol. 5, No. 1, 1967, pp. 51-56.

${ }^{4}$ Donaldson, I. S., "On the Separation of a Supersonic Flow at a Sharp Corner", AIAA Journal, Vol. 5, No. 6, 1967, pp. 1086-1088.

${ }^{5}$ Shang, J. S., and Korkegi, R. H., "Investiation of Flow Separation over a Rearward-Facing Step in a Hypersonic Stream", AIAA Journal, Vol. 6, No. 5, 1968, pp. 986-987.

${ }^{6}$ Wada, I., and Inoue, Y., "Heat Transfer Behind the Backward Facing Step in Hypersonic Flow", In Proceedings of the 10th International Symposium on Space Technology and Science, Vol. 425, 1973, pp. 425-432.

${ }^{7}$ Gai, S. L., Reynolds, N. T., Ross, C., and Baird, J. P., "Measurements of Heat Transfer in Separated High-Enthalpy Disssociated Laminar Hypersonic Flow Behind a Step", Journal of Fluid Mechanics, Vol. 199, 1989, pp. 541-561.

${ }^{8}$ Loth, E., Kailasanath, K., and Löhner, R, "Supersonic Flow over an Axisymmetric Backward-Facing Step", Journal of Spacecraft and Rockets, Vol. 29, No. 3, 1992, pp. 352-359.

${ }^{9}$ Gai, S. L., and Milthorpe, J. F., "Hypersonic High-Enthalpy Flow over a Blunt-Stepped Cone", Proceedings of the 20 th International Symposium on Shock Waves, pp. 234-244, 1995.

${ }^{10}$ Li, H., Moin, P., and Kim, J., "Direct Numerical Simulation of Turbulent Flow over a Backward-Facing Step", Journal of Fluid Mechanics, Vol. 330, 1997, pp. 349-374.

${ }^{11}$ Grotowsky, M. G., and Ballmann J., "Numerical Investigation of Hypersonic Step-Flows", Shock Waves, Vol. 10, 2000, pp. $57-72$.

${ }^{12}$ Hayne, M. J., Gai, S. L., Mee, D. J., and Morgan, R. G., "Real Gas Effects on Flows over Rearward-Facing Steps in High Enthalpy Flows", In Proceedings of the 24th International Symposium on Shock Waves, Beijing, China, 2004.

${ }^{13}$ Leite, P. H. M., and Santos, W. F. N., "Direct Simulation Calculations of the Rarefied Hypersonic Flow past a BackwardFacing Step", 2009 Brazilian Symposium on Aerospace Engineering and Applications, September 14-16, São José dos Campos, SP, Brazil, 2009.

${ }^{14}$ Gai, S. L., and Hayne, M. J., "Heat Transfer Behind a Step in High-Enthalpy Laminar Hypersonic Flow", Journal of Thermophysics and Heat Transfer, Vol. 24, No. 4, 2010, pp. 839-841.

${ }^{15}$ Deepak, N. R., Gai, S. L., and Neely, A. J., "High-Enthalpy Flow over a Rearward-Facing Step - A Computational Study", Journal of Fluid Mechanics, Vol. 695, 2012, pp. 405-438.

${ }^{16}$ Bird, G. A., Molecular Gas Dynamics and the Direct Simulation of Gas Flows, Oxford University Press, Oxford, England, UK, 1994.

${ }^{17}$ Bird, G. A., "Monte Carlo Simulation in an Engineering Context," in Progress in Astronautics and Aeronautics: Rarefied gas Dynamics, edited by Sam S. Fisher, Vol. 74, part I, AIAA New York, 1981, pp. 239-255.

${ }^{18}$ Bird, G. A., "Perception of Numerical Method in Rarefied Gasdynamics, in Rarefied gas Dynamics: Theoretical and Computational Techniques," edited by E. P. Muntz, and D. P. Weaver and D. H. Capbell, Vol. 118, Progress in Astronautics and Aeronautics, AIAA, New York, 1989, pp. 374-395.

${ }^{19}$ Borgnakke, C. and Larsen, P. S., "Statistical Collision Model for Monte Carlo Simulation of Polyatomic Gas Mixture," Journal of computational Physics, Vol. 18, No. 4, 1975, pp. 405-420.

${ }^{20}$ Boyd, I. D., "Analysis of Rotational Nonequilibrium in Standing Shock Waves of Nitrogen," AIAA Journal, Vol. 28, No. 11, 1998, pp. 1997-1999.

${ }^{21}$ Bird, G. A. "A Comparison of Collision Energy-based and Temperature-based Procedures in DSMC," Rarefied Gas Dynamics: 26th International Symposium, edited by T. Abe, American Institute of Physics, 2009, pp. 245-250.

${ }^{22}$ Guo, K., and Liaw, G. S., "A Review: Boundary Conditions for the DSMC Method", In Proceedings of the 35th AIAA Thermophysics Conference, AIAA Paper 2001-2953, 11-14 Jun, Anaheim, CA, 2001.

${ }^{23}$ Alexander, F. J., Garcia, A. L., and, Alder, B. J., "Cell Size Dependence of Transport Coefficient in Stochastic Particle Algorithms", Physics of Fluids, Vol. 10, No. 6, 1998, pp. 1540-1542.

${ }^{24}$ Alexander, F. J., Garcia, A. L., and, Alder, B. J., "Erratum: Cell Size Dependence of Transport Coefficient is Stochastic Particle Algorithms", Physics of Fluids, Vol. 12, No. 3, 2000, pp. 731-731.

${ }^{25}$ Garcia, A. L., and Wagner, W., "Time Step Truncation Error in Direct Simulation Monte Carlo", Physics of Fluids, Vol. 12, No. 10, 2000, pp. 2621-2633. 
${ }^{26}$ Hadjiconstantinou, N. G., "Analysis of Discretization in the Direct Simulation Monte Carlo", Physics of Fluids, Vol. 12, No. 10, 2000, pp. 2634-2638.

${ }^{27}$ Leite, P. H. M., "Direct simulation of the step influence on a reentry vehicle Surface" (in Portuguese), MS Thesis, INPE (National Institute for Space Research), Brazil, 2009. 